\title{
The Management of Keloids: Hands-On Versus Hands-Off
}

\author{
James Studdiford, MD, Amber Stonebouse, MD, Marc Altshuler, MD, and \\ Elliot Rinzler, $M D$
}

Keloids are benign fibrous growths that appear in scar tissue. The lesions can be severely disfiguring
and early recognition of genetic lesions is crucial. This case report outlines and reviews the important
management strategies for these lesions and the requirement for extensive counseling for the patient
and their family. Many potential medical and surgical interventions exist. Unfortunately, these lesions
tend to recur and overall outcomes remain poor. Given patient susceptibility to disfiguring results, sur-
gical intervention should be used with extreme caution. (J Am Board Fam Med 2008;21:149-152.)

\section{Case Presentation}

A 36-year-old African-American man was seen by his family physician for management of morbid obesity and hypertension. Although he was otherwise healthy, he was noted to have marked keloid formation along his jawline and occiput (Figure 1). On further questioning he revealed that he had keloid formation at a very early age; these lesions formed over areas of bony prominence along his shoulder, back, chest, occiput, and ear. These facial lesions remained relatively stable until he began shaving at age 12. At that time he described the development of "shaving bumps," or pseudofolliculitis barbae, which caused ongoing inflammation and added to the keloid formation along his jawline and neck. There was also significant progression in the bulk of the overall keloid covering his ears.

Surgical excision of the keloid tissue along both ears was attempted at age 14, but this debulking procedure exacerbated the problem. This surgical procedure was not preformed at our institution, and the operative and pathology reports were un-

This article was externally peer reviewed.

Submitted 11 January 2007; revised 9 October 2007; accepted 10 October 2007.

From Department of Family and Community Medicine, Thomas Jefferson University Hospital, Philadelphia, PA.

Funding: none.

Conflict of interest: none declared.

Corresponding author: Amber Stonehouse, MD, Thomas Jefferson University Hospital, Family and Community Medicine, 833 Chestnut Street, Suite 301, Philadelphia, PA 19107 (E-mail: amber.stonehouse@mail.tju.edu). available. Soon after the surgery, keloid formation recurred to an even greater degree. At age 18, the patient experienced the formation of new keloids along his occiput and posterior skull (Figure 2). He attributed this phenomenon to trauma associated with participation in high school football competitions. Two years ago, surgical removal of the keloids along his face was again attempted. This time prophylactic radiation therapy was initiated after surgery in an attempt to inhibit fibroblast proliferation and diminish the chance of keloid regrowth. The repeated attempt at debulking was again unsuccessful, and the keloids along his jawline, neck, and ear returned at greater mass. He also revealed a strong family history of keloids, with similar spontaneous formation in several members of his family. A pedigree (Figure 3) illustrates the prevalence and location of keloids in known relatives.

Given this patient's strong family history, he and his family members have since received extensive counseling regarding the risk of future skin trauma, avoidance of elective surgeries, early recognition of infection, and genetic counseling if they are to have children. With the impressive genogram and nature of inheritance in his family, this patient probably has an autosomal dominant trait that would be passed on to his children.

From a psychosocial point of view, the patient admitted to embarrassment and low self-esteem caused by the disfiguring nature of his keloids. $\mathrm{He}$ also reported being the subject of repeated ridicule as a child that left him suffering from depression and anxiety. After extensive counseling from his 


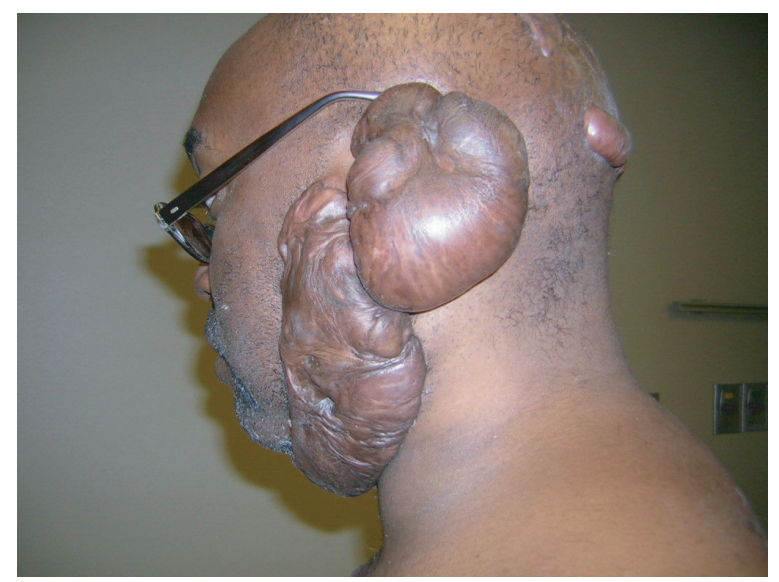

Figure 1. Large bulky keloid formation along the jawline encompassing the ear and extending to the occiput. Seen here after attempted surgical excision with postoperative radiation therapy.

family doctor he became more comfortable with his appearance, entered a supportive relationship, and became gainfully employed.

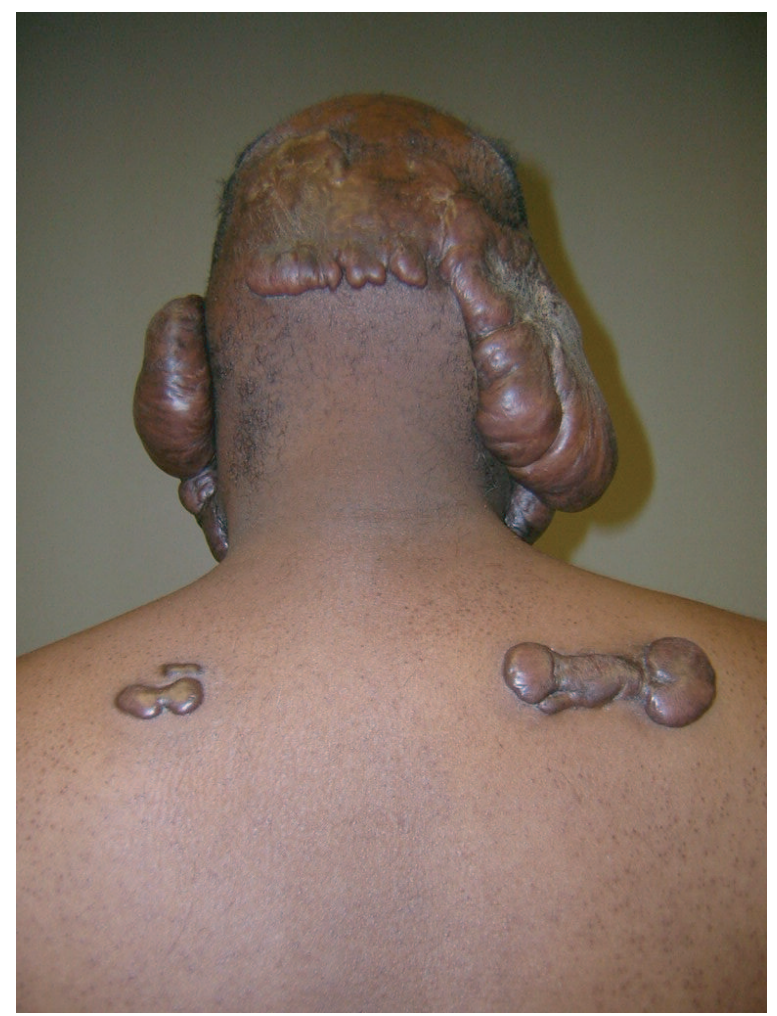

Figure 2. Multiple keloids along the occiput and posterior skull as a result of trauma incurred while wearing a football helmet worn during competitions.

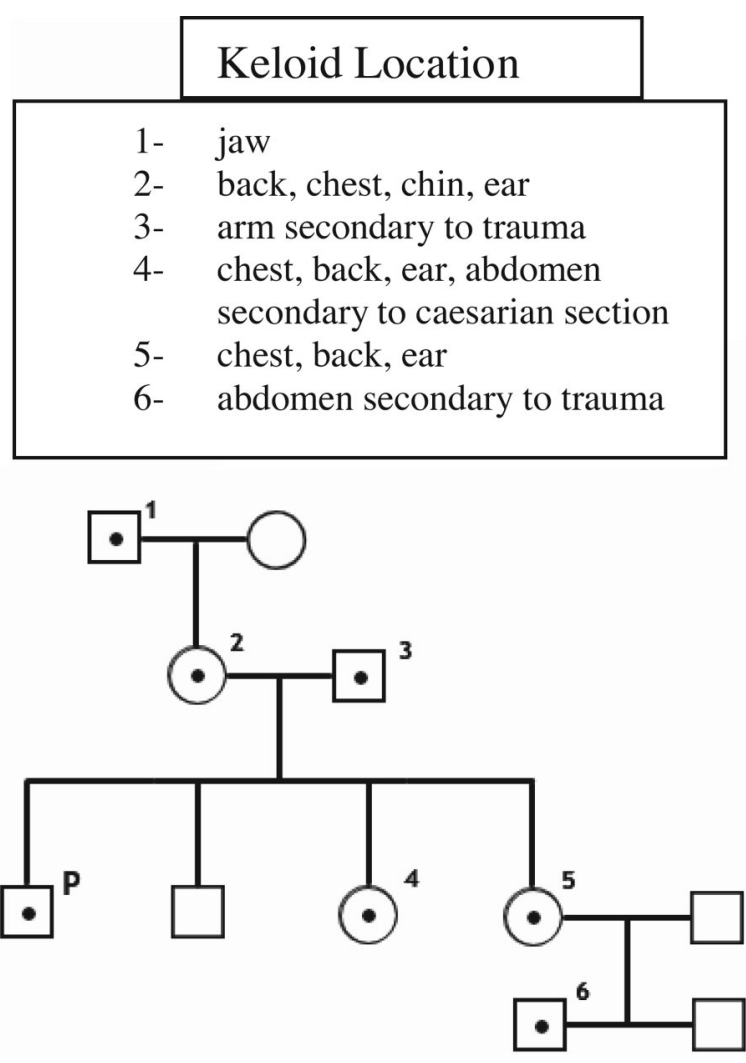

Figure 3. Familial inheritance pattern of keloids.

\section{Discussion}

Keloids develop from a hyperproliferation of dermal fibroblasts and uncontrolled collagen deposition driven by transforming growth factor (TGF)$\beta .^{1} \quad$ These benign fibrous growths represent exuberant scar tissue that extends beyond the confines of the initial trauma. These lesions tend to recur after surgical excision and proliferate in a pseudotumor manner. Keloids are distinguished from hypertrophic scars, which adhere to the border of the original trauma and generally retain their organized configuration. Although the precise pathogenesis is not fully understood, keloids have a well-documented familial predilection, most commonly seen in Asian and African-American populations. ${ }^{2}$ They have been associated genetically with HLA-B14, HLA-B21, HLA-Bw16, HLABw35, HLA-DR5, HLA-DQw3, and blood group A. ${ }^{3}$ In a review by Marneros et $\mathrm{al}^{4}{ }^{4}$ a study of 14 pedigrees with familial keloids, the observed inheritance appeared to be autosomal dominant with incomplete penetrance. Sporadic appearance of keloids does exist, but distinct characteristics between genetic and nongenetic keloids have not been determined. Alterations of apoptosis, cell prolifera- 
tion, and overactivation of insulin-like growth factor have all been implicated in the pathogenesis of keloids. ${ }^{5,6}$ Sayah et $\mathrm{l}^{5}$ compared the expression of 64 apoptosis-related genes in keloids and normal scars. Underexpression of 8 of these genes was found in the fibroblasts derived from keloid tissue. Keloid fibroblasts fail to undergo physiologically programmed cell death and therefore continue to produce connective tissue beyond the period expected for normal scars.

Diagnosis is based on the clinical appearance and location of the scar tissue. Keloids may occur in any location, particularly after trauma, but are seen most commonly on the ears and over bony prominences subject to repeated pressure and friction, such as the sternum and mandible. Although generally asymptomatic, some keloids may be pruritic or tender.

Given the generally poor response to treatment of severe keloids, prevention should be the primary strategy. (Strength of recommendation level C: expert opinion. $)^{7}$ All patients at risk for keloid formation should be counseled to avoid unnecessary trauma (for example, contact football in our patient), body modification, and surgical interventions, including tattoos, body piercing, and cosmetic surgery. Any skin infections (such as folliculitis, pseudofolliculitis, herpes zoster, acne, or furuncles) in predisposed patients should be treated aggressively to minimize inflammation. ${ }^{8}$ Young men need to be instructed in proper management of facial hair. Hair should be trimmed with sharp scissors and cut no shorter than oneeighth of an inch. The family physician should be aware of other modes of prevention for patients with a predisposition to keloid formation, such as the use of semi-occlusive dressings for minor abrasions, splinting after surgical closure of an open wound to avoid excessive tension on the skin, and the use of pressure garments consisting of elasticized material over wounds. Table 1 provides a complete outline of prevention strategies for people predisposed to keloid formation.

For painful or disfiguring keloids, several treatment options exist for minimizing bulk and recurrence (Table 1). A combination of these therapies is most effective, but recurrences are common despite therapy. The earlier keloids are treated, the more likely it is that they will respond to therapy.

Intralesional steroids are the first-line treatment for most keloids: local anesthesia followed by tri-

\section{Table 1. Keloid Prevention and Treatment Strategies}

Prevention strategies for use in people predisposed to keloid formation

1. Pressure garment consisting of elasticized material over wounds.

2. Moist healing by use of dressing impregnated with ointment.

3. Semi-occlusive dressings for minor abrasions.

4. Splinting after surgical closure of an open wound to avoid excessive tension on the skin.

5. Injection of triamciniolone acetonide.

6. Silicon gel dressing for 3 months.

Treatment options for patients with keloids*

1. Intralesional steroid injection

2. Surgical excision

a. Scalpel excision

b. Laser excision

c. Electro excision

3. Cryosurgery

4. 5-flurouracil injection

5. Bleomycin injection

6. Interferon $\alpha$-2b injection

${ }^{*}$ Combination therapy, with one or more of the above treatments, may have greater success rates.

amcinolone 5 to $40 \mathrm{mg} / \mathrm{mL}$ injected into the bulk of the lesion. Injections can be repeated monthly, but treatment should be discontinued if there is no response after 4 injections. Skin atrophy and hypopigmentation may occur at higher doses. Seventy percent of patients respond to intralesional corticosteroid injections with flattening of keloids, although the recurrence rate approaches $50 \%$ at 5 years. $^{\text {? }}$

Scalpel, laser, or electro excision can be considered if injection therapy is unsuccessful. However, these surgical treatments alone result in a recurrence rate of $45 \%$ to $100 \% .{ }^{10}$ Therefore, excision should be used in conjunction with preoperative, intraoperative, and postoperative steroid injection and prophylactic radiation therapy to achieve optimum remission rate.

Cryosurgery alone, for keloids $<6 \mathrm{~mm}$ in depth, has been shown to reduce appearance of keloids in up to $50 \%$ of patients. ${ }^{11,12}$ The major side effect is permanent hypopigmentation, which limits use of cryosurgery in patients with darker skin. Treatment involves a 10 - to 30 -second freeze-thaw cycle repeated up to 3 times per treatment session. Therapy may be repeated once per month until response occurs. 
Several new therapies more specifically directed at intervention and prevention of keloids have recently been investigated. Lypka ${ }^{13}$ found that imiquimod, an immune-modulating medication, effects collagen synthesis in keloids. This study reported that imiquimod significantly suppressed type I collagen expression in keloid fibroblasts. ${ }^{13}$ In another report, Berman et $\mathrm{al}^{11}$ describes the postoperative use of topical imiquimod, which may further reduce the recurrence of keloids. Although more research needs to be done, these findings suggest there may be immune-modulating medications available that could be used in conjunction with surgery to decrease the recurrence of keloids.

Radiation therapy as the sole modality of treatment must be used with caution given the longterm risks of malignancy. This treatment should be reserved for recalcitrant keloids that are resistant to other therapies and are unable to be resected. Adjunctive radiation therapy is highly successful for reducing keloid recurrence, particularly after surgical excision, with reported control rates of $70 \%$ to $90 \% .^{12}$

\section{Conclusion}

For patients with a history of keloids, special care must be taken after traumatic skin injury. Physicians should be aware that the skin should be closed with minimal tension and the use of pressure dressings for 3 to 6 months should be considered to reduce keloid formation. ${ }^{14}$ Treatment options for severe familial keloids, as outlined in this case, are limited and often result in worsening keloid recurrence. The mainstay of therapy should be identification of at-risk individuals and extensive counseling regarding prevention. If need be, a combination of therapies should be used to maximize success rates. Surgical intervention should be used with extreme caution given patient susceptibility to disfiguring outcomes.

\section{References}

1. Slemp A, Kirschner R. Keloids and scars: a review of keloids and scars, their pathogenesis, risk factors, and management. Curr Opin Pediatr 2006;18:396-402.

2. Al-Attar A, Mess S, Thomassen JM, Kauffman CL, Davison SP. Keloid pathogenesis and treatment. Plast Reconstr Surg 2006;117:286-300.

3. Berman B, Perez O, Zell D. Keloid and hypertrophic scar. Updated 1 February 2007. Available at http:// www.emedicine.com/derm/topic205.htm. Accessed 27 October 2006.

4. Maneros A, Norris J, Olsen B, et al. Clinical genetics of familial keloids. Arch Dermatol 2001;137:1429_ 34.

5. Sayah DN, Soo C, Shaw WW, et al. Downregulation of apoptosis-related genes in keloid tissue. J Surg Res 1999;87:209-16.

6. Ohtsuru A, Yoshimoto H, Ishihara H, et al. Insulinlike growth factor-I (IGF-I)/IGF-I receptor axis and increased invasion activity of fibroblasts in keloid. Endocr J 2000;47(suppl):S41-4.

7. Ebell MH, Siwek J, Weiss BD. Strength of Recommendation Taxonomy (SORT): A patient-centered approach to grading evidence in the medical literature. J Am Board Fam Pract 2004;17:59-67.

8. Habif T. Skin disease, 2nd edition. Philadelphia (PA): Elsevier; 2005.

9. Shaffer JJ, Taylor SC, Cook-Bolden F. Keloidal scars: a review with a critical look at therapeutic options. J Am Acad Dermatol 2002;46:S63-97.

10. Berman B, Bieley HC. Adjunct therapies to surgical management of keloids. Dermatol Surg 1996;22: 126-30.

11. Berman B, Villa A. Imiquimod $5 \%$ cream for keloid management. Dermatol Surg. 2003;29:1050-1.

12. Sharad M. Treatment of keloids and hypertrophic scars. Indian J Dermatol Venereol Leprol 2005;71: 3-8.

13. Lypka M. Treatment of keloids with Imiquimod: a possible mechanism via the upregulation of matrixmetalloproteinase-1. J Oral Maxillofac Surg 2006; 64(suppl 1):56-7.

14. Singer AJ, Hollander JE, Quinn JV. Current concepts: evaluation and management of traumatic lacerations. N Engl J Med 1997;337:1142-8. 